\title{
REMARKS ON A PAPER OF HOBBY AND WRIGHT
}

\section{PAUL HILL 1}

C. Hobby and C. R. B. Wright [2] have just published the following Theorem A. However, their proof seems to contain an error. ${ }^{2}$

The notation of [2] is used except that $G_{n}$ is not reserved for the $n$th term of the lower central series of $G: \phi(G)$ denotes the Frattini subgroup of $G ;(G, H)$ means the group generated by the commutators $g^{-1} h^{-1} g h$ where $g \in G, h \in H ;\left(A_{1}, A_{2}, \cdots, A_{n+1}\right)$ is defined inductively as $\left(\left(A_{1}, A_{2}, \cdots, A_{n}\right), A_{n+1}\right) ; H \subset G$ means that $H$ is properly included in $G$.

TheOREM A. If $G$ is a finite p-group and $H$ a subgroup of $G$ such that $H_{n} \subset G_{n}$, then $(H \phi(G))_{n} \subset G_{n}$, where $X_{n}$ denotes the nth term of the lower central series of $X$.

N. Itô [3] had already proved this theorem for the case $n=2$. In this note, Itô's theorem is generalized in a somewhat stronger form than Theorem A. In fact, as was shown in [2], if Theorem A were false, it would have to fail for a normal subgroup $H$ of $G$. In the presence of this fact, Theorem $A$ is contained in

THEOREM B. Let $G_{1} \subseteq G_{2} \subseteq \cdots \subseteq G_{n}=G$ be a nondecreasing finite chain of normal subgroups of a finite p-group $G$ and let $H_{1}, H_{2}, \cdots, H_{n}$ be normal subgroups of $G$ with $H_{i} \subseteq G_{i}$ for all $i$. If

$$
\left(H_{1}, H_{2}, \cdots, H_{n}\right) \subset\left(G_{1}, G_{2}, \cdots, G_{n}\right),
$$

then

$$
\left(H_{1} \phi\left(G_{1}\right), H_{2} \phi\left(G_{2}\right), \cdots, H_{n} \phi\left(G_{n}\right)\right) \subset\left(G_{1}, G_{2}, \cdots, G_{n}\right) .
$$

Proof. Suppose that the theorem is false for a certain $n$. Let $G$ be of minimal order for which it is false and let $H_{1}, H_{2}, \cdots, H_{n}$ be chosen such that if $K_{i}$ is a normal subgroup of $G$ and $H_{i} \subset K_{i} \subseteq G_{i}$ for any $i$, then

$$
\left(H_{1}, H_{2}, \cdots, K_{i}, \cdots, H_{n}\right)=\left(G_{1}, G_{2}, \cdots, G_{n}\right) .
$$

For convenience, set $\left(H_{1}, H_{2}, \cdots, H_{n}\right)=A,\left(H_{1} \phi\left(G_{1}\right),-H_{2} \phi\left(G_{2}\right)\right.$, $\left.\cdots, H_{n} \phi\left(G_{n}\right)\right)=B$, and $\left(G_{1}, G_{2}, \cdots, G_{n}\right)=C$. First, it is noted that

Received by the editors December 19, 1960.

1 This research was supported by the National Science Foundation.

2 The fact that $G$ is noncyclic does not imply that $\phi(G)$ is the intersection of all normal subgroups of index $p^{2}$ in $G$. 


$$
\left(H_{1} / A, \cdots, H_{n} / A\right)=A / A \subset C / A=\left(G_{1} / A, \cdots, G_{n} / A\right) .
$$

Thus if $A \neq\langle 1\rangle$, the relation

$$
B / A=\left(H_{1} \phi\left(G_{1}\right) / A, \cdots, H_{n} \phi\left(G_{n}\right) / A\right) \subset C / A
$$

holds according to the choice of $G$ since in general $\phi(G / N)=N \phi(G) / N$. However, this implies that $B \subset C$; hence $A=\langle 1\rangle$.

Let $z$ be an element of order $p$ in $Z$, the center of $G$. If the relation

$$
\begin{aligned}
\langle z\rangle /\langle z\rangle & =\left(\langle z\rangle H_{1} /\langle z\rangle, \cdots,\langle z\rangle H_{n} /\langle z\rangle\right) \\
& \subset\left(\langle z\rangle G_{1} /\langle z\rangle, \cdots,\langle z\rangle G_{n-1} /\langle z\rangle, G_{n} /\langle z\rangle\right)=\langle z\rangle C /\langle z\rangle
\end{aligned}
$$

holds, then

$$
\langle z\rangle B /\langle z\rangle=\left(\langle z\rangle H_{1} \phi\left(G_{1}\right) /\langle z\rangle, \cdots,\langle z\rangle H_{n} \phi\left(G_{n}\right) /\langle z\rangle\right) \subset\langle z\rangle C /\langle z\rangle,
$$

which implies that $B \subset C$. Thus $C=\langle z\rangle$.

Since all the subgroups involved are normal, from the linearity properties of commutators $[1$, p. 150] it follows that

$$
\begin{aligned}
& \left(H_{1}, \cdots, H_{k-1}, H_{k} \phi\left(G_{k}\right), H_{k+1}, \cdots, H_{n}\right) \\
& \quad=A\left(H_{1}, \cdots, H_{k-1}, \phi\left(G_{k}\right), H_{k+1}, \cdots, H_{n}\right)=\langle 1\rangle .
\end{aligned}
$$

But $H_{k} \subset H_{k} \phi\left(G_{k}\right)$ for some $k$. Thus a contradiction has been established on the choice of the $H_{i}$, and the theorem is proved.

\section{REFERENCES}

1. M. Hall, The theory of groups, New York, Macmillan, 1959.

2. C. Hobby and C. R. B. Wright, $A$ generalization of a theorem of $N$. Ito on $p$ groups, Proc. Amer. Math. Soc. vol. 11 (1960) pp. 707-709.

3. N. Ito, On a theorem of $L$. Redei and J. Szép concerning p-groups, Acta Sci. Math. Szeged. vol. 14 (1952) pp. 186-187.

The Institute for Advanced Study 